\title{
Real Time Iris Image Segmentation for Non Co-Operative Environment
}

\author{
Patil Mayur J. \\ Assistant Professor \\ Department of Computer Engineering \\ SSVPS B.S.Deore College of Engineering \\ Dhule,India
}

\author{
K. P. Adhiya \\ Associate Professor \\ Department of Computer Engineering \\ SSBT's College of Engineering \& Technology \\ Bambhori,Jalgaon,India
}

\begin{abstract}
In the area of iris recognition, the most difficult \& complex part is iris segmentation. There are several different algorithms proposed \& developed in recognition area. Iris recognition becomes more difficult in case of non co-operative environment. Most of the time due to some condition or situation an eye changes with respect to lens of camera, size, shape \& iris pattern details will change as well as it is very difficult to match these samples with enrolled images using traditional method. The traditional algorithm can suitable for frontal iris images. In non co-operative environment there is no consistent location of eye in the image. Sometimes, the eye may have closed or blinking without a proper iris pattern or there is no eye in the image. When such situation occurs, the result of traditional iris image recognition method would dramatically decrease. So the real time image processing provides better information as compare to traditional method \& it is proper solution as compare to processing a single image.
\end{abstract}

\section{General Terms:}

Segmentation, Recognition

Keywords:

K-Mean, PCA, ICA, Iris, Pattern Matching

\section{INTRODUCTION}

Individual \& accurate personal recognition is more difficult \& complex for many recognition system. Today, each \& every organization uses such type of system which gives very effective result in minimal time. Due to the security \& reliability issue each \& every organization keeps the record of their individual worker. So achieving better reliability \& perfect result the demand of biometric system is increases day by day. The basic aim of biometric system is to give an identity based on who we are rather than on what we possesses or what we remember. Pattern recognition is one of the key processes which have been used by human for last so many years. Pattern recognition means collecting or capturing raw data $\&$ produces the result according to pattern. In the area of computer science, the pattern recognition system plays a vital role to solve our daily problems [17].
Figure 1 shows the basic form of the pattern recognition system. Pattern recognition system can be divided into preprocessing, feature extraction \& classification. First, the raw data is collected which is used as sample data for all the remaining processes. This process is basically known as data capture process [17].

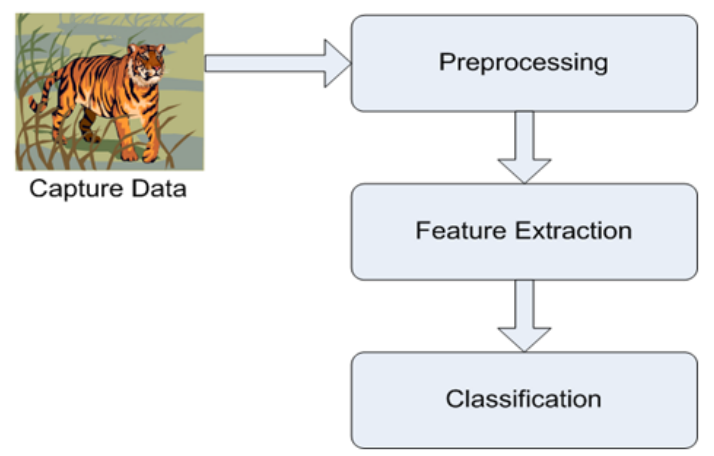

Fig. 1. Basic Form of Pattern Recognition System [17]

Next phase is preprocessed phase, which simply removes the noise i.e. the data which is not required. It is very complex phase; due to this the preprocessing phase is subdivided into certain modules. Segmentation is one of them. In segmentation the basic focus on isolation of the region of sample data \& localization of each data component. It is very basic stage of the any pattern recognition system. It is directly interacting with data capturing device \& the conditions. The normalization is one of the important phases in the pattern recognition system. The feature extraction field deals with statistical representation of data. It characterized data by using several features. It is useful to detect irrelevant transformation of input data. This phase required proper guideline from expert person in a particular knowledge area \& it greatly find out the correctness of the system. The final stage is classification, which takes the data from feature extraction phase \& generates a proper decision or set of class. The classification phase can be made up of three different approaches. The first is simplest \& based on the concept of similarity. One of the best example of this is template making. The second one is statistical. The basic assumption of this is the data \& patterns are generated by the probabilistic system. The last approach 
is decision boundaries through the optimization of some error criterion [17].

\subsection{Structure of an Iris}

An iris makes the pupil wider \& narrower to regulate the amount of light which enter in the eye \& which affect on the retina. An iris consists of stroma \& epithelial layer, out of which the stroma is anterior part \& epithelial layer is posterior part. Figure 2 shows the pupillary zone \& ciliary zone which are separated by the collarette. These two zones contain different textural information [1].

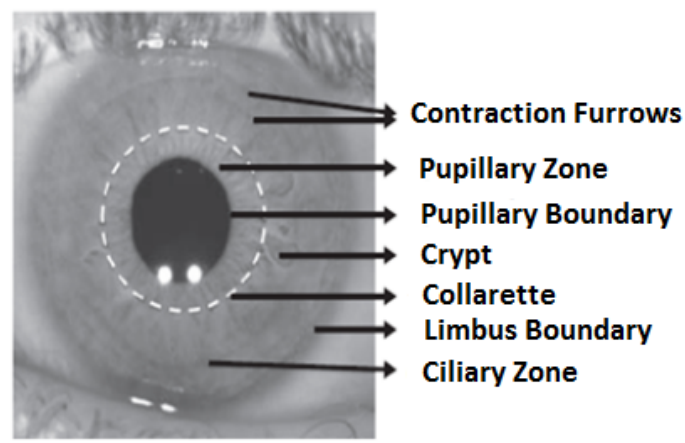

Fig. 2. Structure of an Iris [1]

There are two boundary areas in an iris such as limbus boundary \& pupillary boundary; both are define the spatial extent of an iris \& in 2D images of eye, help to describes it in detail from other ocular structure like eyelashes, eyelids, sclera pupil. The rich textural information is in the iris frontal surface which provides a powerful biometric proof for human identification [1]. Figure 3 \& Figure 5 shows transverse section $\&$ frontal section of an iris. For any iris detection system it is very important to understand the structure of an iris before proceeding to perform iris detection [19].

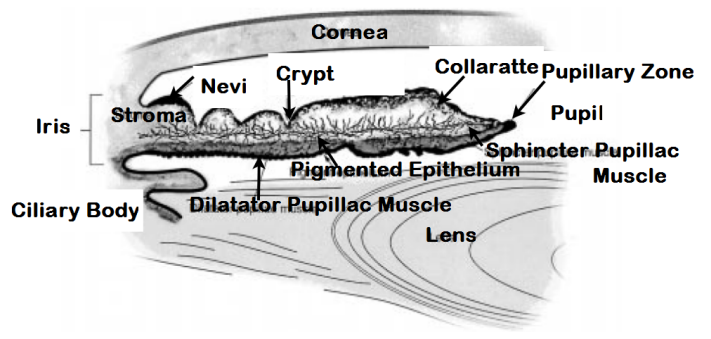

Fig. 3. Structure of Iris seen in transverse section [19]

Iris structure is divided into several layers such as,

(1) Pigmented Epithelial Cell Layer

(2) Stromal Layer

(3) Chromataphores

This multilayer structure is responsible for visual appearance of an iris. The color of Iris is due to differential absorption of light falling on the pigmented cell [19].

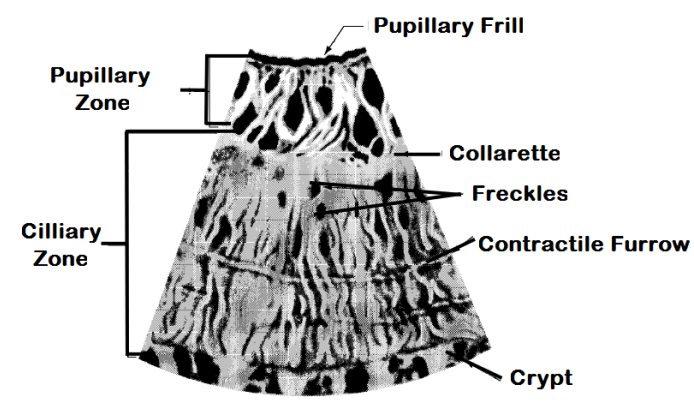

Fig. 4. Structure of Iris seen in frontal section

\section{SEGMENTATION METHODOLOGIES}

From the Greek word "bio"\& "metrics"the term "Biometric"is derived. In Greek "bio"means life \& "metrics"means to measure. There are so many advantages of the automated biometric system in the computer processing area. Many automated techniques are based on ideas that were come from hundreds, even thousands of years ago [2]. For proper functioning of the society, human identification is the best way \& it plays very important role around the society. There are so many different ways to identify human such as voice, appearance \& gait for thousands of year. In $19^{t h}$ century Alphonse Bertillon introduced the use of number of anthropomorphic measurement to identify habitual criminal. This is one of the systematic \& scientific ways for human identification. After this in early 1900's fingerprint is used in forensic investigation to identify the criminals \& suspects. Nowadays, fingerprint identification system is used worldwide by all law enforcement agencies. Other physiological \& behavioral characteristics have been used to identify the human because of increasing growth in terrorist activity, security breaches \& financial fraud. There are different biometric characteristics used for person identification which includes fingerprints, palm, iris, face \& voice. Biometric is one of the important techniques that are used worldwide in variety of applications [12]. Flom \& Safir in 1987 presented a very first relevant method. There are so many distinct approaches have been proposed [8].

Daugman in 1993, introduced an integro differential operators. This operator is able to find out the inner \& outer border of an iris. After some minor differences this operator was again proposed by Nishino \& Nayar in 2004 [6].

Camus \& Wildes [5] \& Martin Roche et al [18] also proposed an integro differential operator which search over an $\mathrm{N}^{3}$ space. This method maximize the equation that identify the iris border.

Wildes proposed a very common method for iris segmentation through gradient based binary edge map construction followed by circular hough transform [21].

On the basis of threshold \& function maximization Liam et al proposed a very simple method for segmentation. This particular method obtain two ring parameters corresponding to the iris inner \& outer borders [14].

On the basis of prior pupil identification an iris detection method have proposed by Du et al. In this method the iris image is converted into polar coordinates $\&$ the outer border of an iris is identified as the largest horizontal edge resultant from sobel filtering. But this particular approach is not work well in non cooperative iris \& pupil also in very dark iris image [7].

Mira \& Mayer use morphologic operator for finding an iris border. For detecting inner border of an iris they apply threshold \& image 
opening \& closing \& for detecting outer border they apply image threshold \& closing \& opening sequences.

By using expectation maximization algorithm Kim et al proposed a method to estimates the respective distribution parameters. In this method they shows that dark, intermediate \& bright distributions contains the pixel corresponding to the pupil, iris \& reflection areas. Kim et al proposed this method on the basis of assumptions that the image captured intensity values can be well represented by a mixture of three gaussian distribution components.

There are two major strategies for iris segmentation first one is boundary based approach \& another one os template based approach. In boundary based strategies some of the geometric algorithms are used for constructing an edge map of an iris \& in template based strategy maximization of some equations are involved. The Wildes method comes under boundary based strategy \& Daugman's, Camus \& Wildes \& Martin-Rotche methods are comes under template based strategy [16]. Following section describe very basic information about these four method.

\subsection{Daugman's Method}

In this method Daugman use an integro differential operator \& assumed that pupil \& iris is in circular form, equ. 1 shows daugman integro differential operator,

$$
\max \left(r, x_{0}, y_{0}\right)\left|G_{\sigma}(r) * \frac{\delta}{\delta r} \oint_{r, x_{0}, y_{0}} \frac{I(x, y)}{2 \pi r} d s\right|
$$

where, $\mathrm{x}_{0}, \mathrm{y}_{0}$ be the center coordinates, $\mathrm{G}_{\sigma}(\mathrm{r})$ be a Gaussian Kernal, $\mathrm{ds}$ be the circular arc, $\mathrm{r}$ be the radius, (x,y) be the image domain. Basically this method searches in an $\mathrm{N}^{3}$ space for the circumference center \& radius that have the highest derivative value when compared with that of neighbor radius [16].

\subsection{Wildes Method}

Wildes proposed this method in 1997 which performs contour fitting process in two steps. First step converts the image intensity information into binary edge map. In second step, the edge points vote to instantiate particular contour parameter values [16].

In first step, gradient based canny edge detector is used to construct the edge map \& in second step, circular Hough transform where each edge point votes for particular contour parameter values [16]. Following equation is used for analyzing the image to estimates the three parameters of one circle $(\mathrm{x}, \mathrm{y}, \mathrm{r})[4]$.

$$
H\left(x_{0}, y_{0}, r\right)=\sum_{i} h\left(x_{i}, y_{i}, x_{0}, y_{0}, r\right)
$$

Where, $\mathrm{x}_{i}, \mathrm{y}_{i}$ is an edge pixel \& $\mathrm{i}$ is the index of edge pixel [4].

$$
h\left(x_{i}, y_{i}, x_{0}, y_{0}, r\right)=\left\{\begin{array}{l}
1 \text { ifg }\left(x_{i}, y_{i}, x_{0}, y_{0}, r\right)=0 \\
0 \text { Otherwise }
\end{array}\right.
$$

Where

$$
g\left(x_{i}, y_{i}, x_{0}, y_{0}, r\right)=\left(x_{i}-x_{0}\right)^{2}-\left(y_{i}-y_{0}\right)^{2}-r^{2}
$$

Edge detection \& Hough Transform uses threshold values for edge detection, because of this the result of hough transform is significantly different [4].

\subsection{Camus and Wildes Method}

In 2004, Camus and Wildes proposed a method which finds person's iris in a closed-up image. This method searches in $\mathrm{N}^{3}$ space as like Daugmans methodology for three circumference parameters by maximizing the functions [4].

$$
C=\sum_{\theta=1}^{n}\left((n-1)\left\|g_{\theta, r}\right\|-\sum_{\phi=\theta-1}^{n}\left\|g_{\theta, r}-g_{\phi, r}\right\|-\frac{I_{\theta, r}}{n}\right)
$$

where $\mathrm{n}$ is the total number of directions \& $\mathrm{I}_{\theta, r}$ image intensity with respect to radius $\& \mathbf{g}_{\theta, r}$ is the image derivative with respect to radius $\mathrm{r}$ in polar coordinate system [16].

This method is properly works on the image which does not contains any reflections or which does not contains any noisy factor. Also this method is work very correctly where the pupil \& iris regions intensities are clearly separated from the sclera [4].

\subsection{Martin-Roche Methods}

Martin proposed this methodology which is very much similar to the Daugman's methodology. In this method Martin uses gray scale image \& on that grey scale image Martin applies a histogram stretch. By using histogram stretch Martin tries to maximize the average intensity differences of the five consecutive circumferences [4], which is defined as

$$
D=\sum_{m}\left(\sum_{k=1}^{5}\left(I_{n, m}-I_{n-k, m}\right)\right)
$$

Where $\mathrm{I}_{i, j}=\mathrm{I}\left(\mathrm{x}_{0}+\mathrm{i} \Delta_{r} \cos \left(\mathrm{j} \Delta_{\theta}\right), \mathrm{y}_{0}+\mathrm{I} \Delta_{r} \sin \left(\mathrm{j} \Delta_{\theta}\right)\right)$.

$\Delta_{r}$ is the radius increments \& $\Delta_{\theta}$ is the angle increments, \& The image intensity is represented by $\mathrm{I}(\mathrm{X}, \mathrm{Y})[4]$.

Practically, this method is very useful to find out the three $\mathrm{N}^{3}$ space circumference parameters (center (x, y) and radius $r$ ), where the intensity difference between five successive circumferences is maximal [4].

\section{PROPOSED SYSTEM}

In above section we covered the basic information about biometric system \& four segmentation method which is most commonly used in any iris recognition system. In this section we discuss how the proposed system work in non co-operative environment. Basically the proposed system is categorized into two section, first section covers segmentation part \& second section covers recognition part. In proposed system K-Mean Clustering algorithm is used for segmentation purpose \& for recognition Principal Component Analysis (PCA) \& Independent Component Analysis (ICA) algorithms are used. In following subsection we cover how the proposed system is work.

\subsection{K-Mean Clustering}

A most common clustering algorithm used in the area of iris recognition \& segmentation is K-Mean Clustering algorithm. K-mean is nothing but partitioning clustering approach. In K-mean data sets are partitions iteratively. In K-mean clustering " $\mathrm{K}$ " is for number of cluster \& this algorithm takes an input from user. K-mean is an unsupervised learning method. From a set of particular data points the $\mathrm{K}$-means classify that data points into number of K-cluster. K-mean is very simple \& understandable clustering algorithm. In K-mean clustering items are automatically assigned to clusters.

The process which groups similar objects from given data set is known as Clustering Process [13]. Another definition of clustering is placing the similar objects into one cluster \& placing dissimilar 
object into another cluster. There are two types of learning method one is supervised learning \& another is unsupervised learning. Kmean belongs to unsupervised learning method. In unsupervised learning there is no need to supervise the given data set.

Without user specified parameter K-mean is not work. The user specified parameter contains total number of clusters, cluster initialization \& distance metric. For clustering large set of data KMean clustering is widely used. One of the drawback of k-mean is it required predefine parameter. So by giving wrong number of $\mathrm{K}$ cluster, the result is not proper. Often, the clustering algorithm required different values of $\mathrm{k}$ to run the program, so for this the best value of $\mathrm{K}$ is select using predefine criteria. For any clustering algorithm stability is most widely used tool for selection of model, in to choose number of cluster $\mathrm{K}$ [13].

The main aim of K-mean Clustering is to minimize an objective functions. The objective function is defined as in a following way [20],

$$
J=\sum_{j=1}^{k} \sum_{i=1}^{n}\left\|x_{i}^{(j)}-c_{j}\right\|^{2}
$$

Where $\left\|\mathrm{x}_{i}{ }^{(j)}-\mathrm{c}_{j}\right\|^{2}$ is a chosen distance measure between a data point $\mathrm{x}_{i}{ }^{(j)}$ and the cluster center $\mathrm{c}_{j}$ is an indicator of the distance of the $\mathrm{n}$ data points from their respective cluster centers [20]. The objective function is also called as cost function ${ }^{1}$

Figure 5 shows work flow diagram of Proposed System,

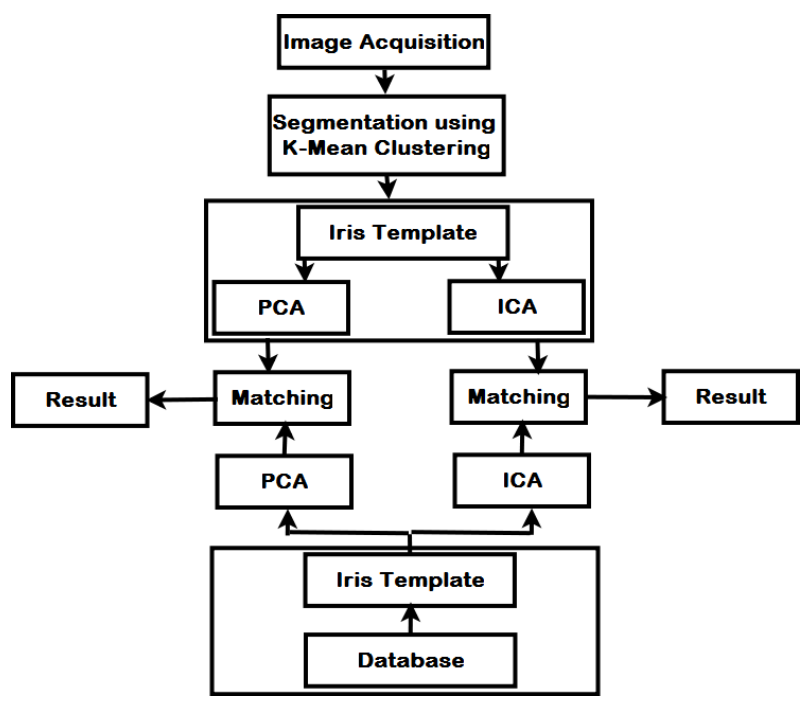

Fig. 5. Work Flow of Proposed System

Following figure shows the segmented images of proposed system. This images capture in non co-operative environment.

Most of the researchers select K-Mean clustering algorithm for their research because of following reasons [3],

(1) Time Complexity $\rightarrow$ The time complexity of K-Mean Clustering Algorithm is $\boldsymbol{o}(\boldsymbol{n k l})$ [3]

Where $\mathrm{n}$ is the number of pattern.

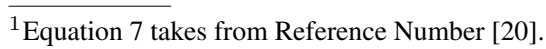

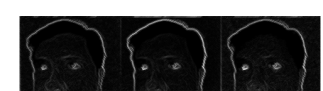

(a)

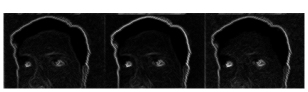

(c)

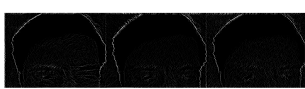

(e)

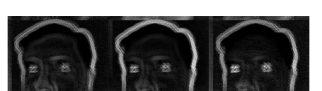

(b)

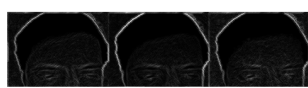

(d)

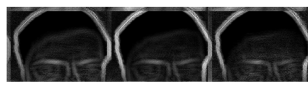

(f)
Fig. 6. Segmented Images generated by Proposed System (a), (b), (c) for PCA \& (d), (e), (f) for ICA

$\mathrm{k}$ is the number of cluster.

1 is the number of iteration taken by an algorithm to converge.

(2) Space Complexity $\rightarrow$ The space complexity of K-Mean Clustering Algorithm is $\boldsymbol{o}(\boldsymbol{k}+\boldsymbol{n})$. For storing data matrix it required additional space [3].

(3) Order Independent $\rightarrow$ Generates the same partition of the data irrespective of the order in which the patterns are presented to the algorithm [3].

\subsection{Principal Component Analysis (PCA)}

PCA is used to find out similarity \& dissimilarity in the given data set $\&$ is also used to find out the data pattern. The aim of PCA is feature extraction from the given database. Feature extraction is one of the important parts of an iris recognition system in which unwanted data is removed. PCA is basically used on huge data set i.e. multivariate data set. The aim of PCA is to extract useful information from multivariate data set.

For multivariate data set the old \& best technique is Principal Component Analysis. In 1901, Pearson introduced this technique \& in 1933, Hotelling developed this technique independently. PCA is useful when the user want to extract useful data from the multivariate data set. PCA is based on reduced dimensionality. Therefore, trends in multivariate data are easily visualized.

Principal Component Analysis(PCA) is depend on two terminology i.g Eigen Vector \& Eigen Value. Basically, Eigenvector provides the weights to compute the uncorrelated PC, which are the linear combination of the centered standardized or centered un-standardized original variables. Eigenvalues measure the amount of the variation explained by each PC and will be largest for the first PC and smaller for the subsequent PCs. Figure 7 shows working of Principal Component Analysis.

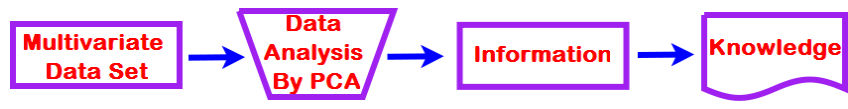

Fig. 7. General Concept of Principal Component Analysis 
PCA does not disregard covariances and correlations, it concentrates on variances. The first step is to look for a linear function $\alpha_{1}^{\prime} \mathrm{X}$ of the elements of $\mathrm{X}$ having maximum variance, where $\alpha_{1}$ is a vector of $P$ constants $\alpha_{11}, \alpha_{12}, \ldots . ., \alpha_{1 p} \&$ denotes the transpose [11], so that

$$
\alpha_{1}^{\prime} X=\alpha_{11} x_{1}+\alpha_{12} x_{2}+\ldots . .+\alpha_{1 p} x_{p}=\sum_{j=1}^{p} \alpha_{1 j} x_{j}
$$

Figure 8 gives a plot of 50 observations on two highly correlated variables $\mathrm{x}_{1}, \mathrm{x}_{2}$. There is considerable variation in both variables, though rather more in the direction of $\mathrm{x}_{2}$ than $\mathrm{x}_{1}$ [11].

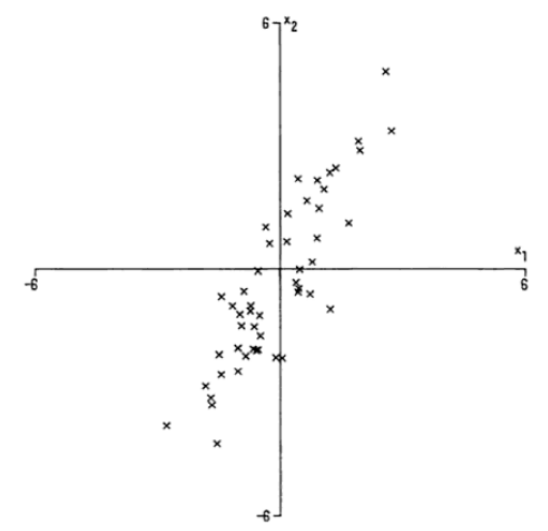

Fig. 8. 50 observations on two variables $\mathrm{x}_{1}, \mathrm{x}_{2}$

Next, look for a linear function $\alpha_{2}^{\prime} \mathrm{X}$ which is uncorrelated with $\alpha_{1}^{\prime} \mathrm{X}$ and has the maximum variance, and so on, so that in the $\mathrm{k}^{t h}$ stage a linear function $\alpha_{k}^{\prime} \mathrm{X}$ is found that maximum variance subject to being uncorrelated with $\alpha_{1}^{\prime} \mathrm{X}, \alpha_{2}^{\prime} \mathrm{X}, \ldots . ., \alpha_{k-1}^{\prime} \mathrm{X}$. The $\mathrm{k}^{\text {th }}$ derived variable, $\alpha_{k}^{\prime} \mathrm{X}$ is the $\mathrm{k}^{\text {th }} \mathrm{PC}[11]$.

Up to $P$ PCs could be found, but in general, most of the variation in $X$ will be accounted for by $m$ PCs, where $m<<P$ [11].

Let us consider a simple case where $P=2$. The advantage of $P=2$ is, the data can be plotted exactly in two dimensions. Figure 8 gives a plot of 50 observations on two highly correlated variables $\mathrm{x}_{1}$, $\mathrm{x}_{2}$. There is considerable variation in both variables, though rather more in the direction of $\mathrm{x}_{2}$ than $\mathrm{x}_{1}$. If we transform to PCs $\mathrm{z}_{1}, \mathrm{z}_{2}$ we obtain the plot given in Figure 9 [11].

It is clear that there is a greater variation in the direction of $z_{1}$ than in either of the original variables, but there is a very little variation in the direction of $z_{2}$. Generally, if a set of $P(>2)$ variables has considerable correlations between them, then the first few PCs will explain most of the variation in the original variables. On the other hand, the last few PCs determine directions in which there is small variation; In other words, they identify near-constant linear correlations among the original variables [11].

\subsection{Independent Component Analysis (ICA)}

For finding hidden factors that underlie sets of random variables, measurements, or signals, there is a statistical and computational technique used which is known as Independent Component Analysis (ICA). For large set of database, ICA defines generative model which observed multivariate data. this model assumes the data variables as to be linear mixture of some latent variable \& the mixing

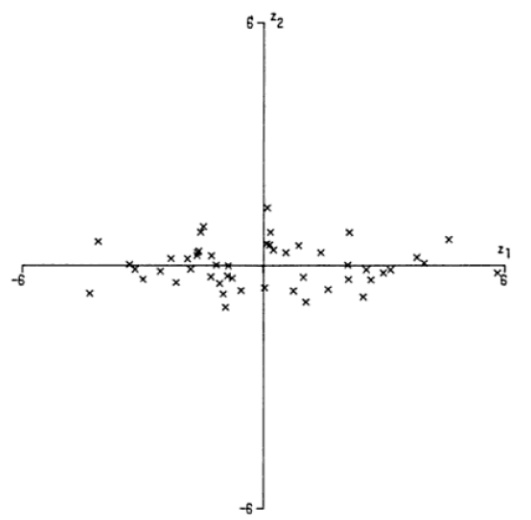

Fig. 9. 50 observations from Figure 8 with respect to their PCs $\mathrm{z}_{1}, \mathrm{z}_{2}$,11]

system is also unknown. These latent variables are non gaussian \& mutually independent $\&$ hence they are called as independent variable of that observed data. ICA is directly related with principal component analysis \& factor analysis. The data analyzed by ICA originate from different application fields such as digital images, document databases, economic indicators and psychometric measurements [9].

Often the measurement are in the form of set of parallel signals or time series, for this blind source separation is used to solved this problem. A very basic application of ICA is "Cocktail Party Problem". Where the underlying speech signals are separated from a sample data consisting of people talking simultaneously in a room. If there is $\mathrm{N}$ number of sources are present, at least $\mathrm{N}$ observations are needed to get the original signal. By maximizing the statistical independence of the estimated components, ICA finds the independent components [9].

To find a linear representation of non gaussian data, Independent Component Analysis is used \& because of this the components are statistically independent, or as independent as possible. This type of representation capture the structure of data in many applications, including feature extraction and signal separation [9].

A statistical latent variable model is used to define independent Component Analysis. Suppose there is $n$ linear mixtures $\mathrm{x}_{1}, \mathrm{x}_{2}$, ....., $\mathrm{x}_{n}$ of $n$ independent component [10]

$$
x_{j}=a_{j 1} s_{1}+a_{j 2} s_{2}+\ldots . .+a_{j n} s_{n}, \quad \text { for all } j
$$

Here time index $t$ is dropped. In the ICA model assume that each mixture $\mathrm{x}_{j} \&$ independent component $\mathrm{s}_{k}$ is a random variable, instead of proper time signal. The observed values $\mathrm{x}_{j}(t)$ are then a sample of this random variable. Assume that both the mixture variables and the independent components have zero mean. If this is not true, then the observable variables $\mathrm{x}_{i}$ can always be centered by subtracting the sample mean, which makes the model zeromean [10].

Let,

$\mathbf{x} \rightarrow$ the random vector whose elements are the mixtures $\mathrm{x}_{1}, \mathrm{x}_{2}$, $\ldots ., \mathbf{x}_{n}$.

$\mathbf{s} \rightarrow$ random vector with elements $\mathrm{s}_{1}, \mathrm{~s}_{2}, \ldots ., \mathrm{s}_{n}$.

$\mathbf{A} \rightarrow$ the matrix with elements $\mathrm{a}_{i j}$.

These all are column vector; so $\mathbf{x}^{T}$, or transpose of $\mathbf{x}$, is a row vector. So using all this notation the mixing model is as shown below [10], 


$$
x=A s
$$

By using columns of matrix $\mathbf{A}$; which is denoted by $\mathrm{a}_{j}$, so the model becomes,

$$
x=\sum_{i=1}^{n} a_{i} s_{i}
$$

Equation 10 is a statistical model \& this model is called as Independent Component Analysis or ICA Model. It describes how the observed data are generated by a process of mixing the components $\mathrm{s}_{i} \&$ hence ICA is called as generative model. The independent components cannot be directly observed, because they are latent variable. Also the mixing matrix is assumed to be unknown [10]. ICA is related with blind source separation (BSS) or blind signal separation method. A "source"means an original signal, i.e. independent component, like the speaker in a cocktail party problem. "Blind"means that we no very little, if anything, on the mixing matrix, and make little assumptions on the source signals. ICA is one method, perhaps the most widely used, for performing blind source separation [10]. Figure 10 shows block diagram of Blind Source Separation Method [15].

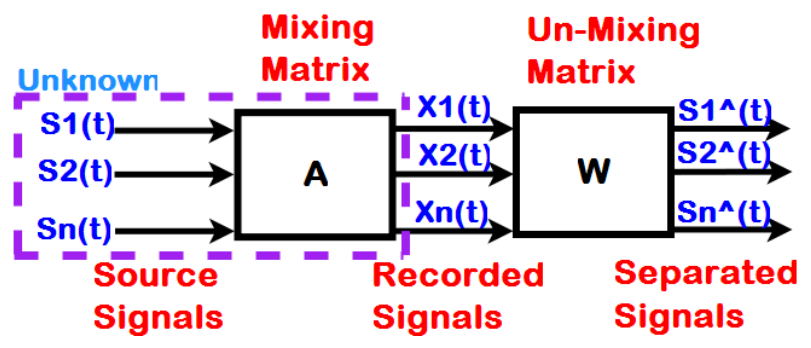

Fig. 10. Blind Source Separation Methods Block Diagram

ICA is a very general-purpose statistical technique in which observed random data are linearly transformed into components that are maximally independent from each other, and simultaneously have "interesting"distributions. ICA can be formulated as the estimation of a latent variable model. The intuitive notion of maximum non gaussianity can be used to derive different objective functions whose optimization enables the estimation of the ICA model. Alternatively, one may use more classical notions like maximum likelihood estimation or minimization of mutual information to estimate ICA; somewhat surprisingly, these approaches are (approximatively) equivalent [10].

\section{EXPERIMENTAL RESULT}

This section focuses on the performance of both recognition algorithm i.e. PCA \& ICA. Table 1 shows the matching result of PCA algorithm. As shown in table, using PCA algorithm the result of recognition system is almost above $90 \%$ \& the average matching result in percentage is up to $93.30 \%$.

Table 2 shows matching result of ICA algorithm. As shown in table, using ICA algorithm the result of recognition system is almost below $90 \%$ \& the average matching result in percentage is up to $88.89 \%$.

Basically there are 50 different real time iris images is used to check the accuracy of proposed system $\&$ the system gives more accurate
Table 1. Matching Result of PCA

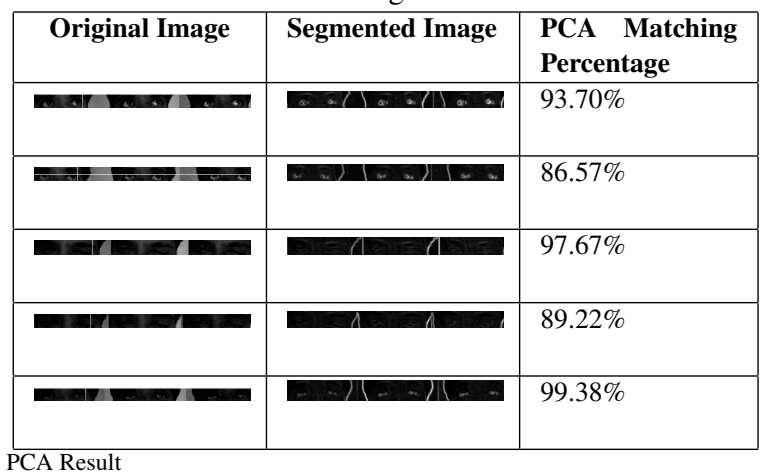

\begin{tabular}{|c|c|c|}
\hline Original Image & Segmented Image & $\begin{array}{l}\text { ICA Matching } \\
\text { Percentage }\end{array}$ \\
\hline mar was wa & & $89.76 \%$ \\
\hline$=$ & & $85.43 \%$ \\
\hline$=1$ & & $87.98 \%$ \\
\hline 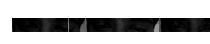 & & $90.39 \%$ \\
\hline 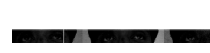 & 11 & $90.91 \%$ \\
\hline
\end{tabular}

Table 2. Matching Result of ICA

matching percentage for PCA then ICA. All of these images takes in non co-operative environment to check the better performance \& accuracy of proposed system.

\section{CONCLUSION}

This paper compares, the performance of two recognition algorithm i.e. Principal Component Analysis \& Independent Component Analysis. Nowadays, need of secure authentication system is increasing worldwide. So this paper gives the basic idea about how the recognition algorithm works. Basically PCA is more accurate then ICA, because PCA totaly work on eigen value \& eigen vector. In co-operative environment it is easy to find out the accuracy of an Iris but in non co-operative environment it is quite difficult task, because in non co-operative environment the subject does not pay attention to the camera \& due to this iris images are blurred, not cleared etc. This paper shows that in non co-operative environment both algorithm (PCA \& ICA) work very well but the accuracy $\&$ performance of PCA is high as compared to ICA. So Principal Component Analysis is the best method for iris recognition system in co-operative as well as non co-operative environment.

\section{REFERENCES}

[1] Biometric history. August 2006.

[2] Iris recognition. August 2006.

[3] O. A. Abbas. Comparisons between data clustering algorithms. The International Arab Journal of Information Technology, 5(3):320-325, July 2008. 
[4] B.O.Adegoke, E.O.Omidiora, S.A.Falohun, and J.A.Ojo. Iris segmentation: a survey. International Journal of Modern Engineering Research (IJMER), 3(4):1885-1889, Jul - Aug. 2013.

[5] T. Camus and R Wildes. Reliable \& fast eye finding in closeup images. IEEE $16^{\text {th }}$ International Conference on Pattern Recognition, Quebec, Canada, pages 389-394, 2004.

[6] J. Daugman. High confidence visual recognition of person by a test of statistical independence. IEEE Trans. Pattern Anal. Mach. Intell., 15(11):1148-1161, 1993.

[7] Y. Du, R. Ives, D. Etter, T. Welch, and C. Chang. A new approach to iris pattern recognition. SPIE Europian Symp. on Optics/Photonic in Defence and Security, London, UK, October 2004.

[8] L. Flom and A. Safir. Iris recognition system. U.S. Patent 4641394, 1987.

[9] K. Gulmire and S. Ganorkar. Iris recognition using independent component analysis. International Journal of Emerging Technology and Advanced Engineering, 2(7):433-437, July 2012.

[10] A. Hyvarinen and E. Oja. Independent component analysis: Algorithms and applications. Neural Networks, 13(4):411430, 2000.

[11] I.T.Jolliffe. Principal Component Analysis,. Springer, 2 edition, April 2002.

[12] Anil K. Jain and Ajay Kumar. Biometrics of next generation: An overview. Second Generation Biometrics, Springer, 2010.

[13] G. Komarasamy and A. Wahi. An optimized k-means clustering technique using bat algorithm. European Journal of Scientific Research, 84(2):263-273, 2012.

[14] L. Liam, A. Chekima, L. Fan, and J. Dargham. Iris recognition using self orgnizing neural network. IEEE 2002 Students Conference on Research \& Developing System, Malaysia, pages 169-172, 2002.

[15] G. R. Naik and D. K. Kumar. An overview of independent component analysis and its applications. Informatica, 35:63$81,2011$.

[16] H. Proenca and L.A. Alexandre. Iris segmentation methodology for non co-operative recognition. IEE Proceedings Vision, Image \& Signal Processing, 153(2):199-205, April 2006.

[17] Hugo Pedro Martins Carrico Proenca. Towards Non Cooperative Biometric Iris Recognition. $\mathrm{PhD}$ thesis, Department of Computer Science, University of Beira Interior, Covilha,Portugal, October 2006.

[18] Martin Roche, C. Sanchez Avila, and R. Sanchez Reillo. Iris recognition for biometric identification using dyadic wavelet transform zero crossing. IEEE Aerosp. Electronics System Mag., 17(10):3-6, 2002.

[19] Dhananjay Theckedath. Iris detection based on principal component analysis-eigen irises. Proceedings of SPIT-IEEE Colloquium and International Conference, Mumbai, India, 1:49_ 52, 2007-2008.

[20] T. Velmurugan and T. Santhanam. Performance evaluation of $\mathrm{k}$-means and fuzzy c-means clustering algorithms for statistical distributions of input data points. European Journal of Scientific Research, 46(3):320-330, 2010.

[21] R. Wildes. Iris recognition: An emerging biometric technology. IEEE Proceding, 85(9):1348-1363, 1997. 